\title{
The stability of a horizontal interface between air and an insulating liquid subjected to charge injection
}

\author{
Rafael Chicón ${ }^{1, a)}$ and Alberto T. Pérez ${ }^{2, b)}$ \\ ${ }^{1}$ Departamento de Electromagnetismo y Electrónica, Universidad de Murcia, Apdo. 4021, \\ 30071 Murcia, Spain \\ ${ }^{2}$ Departamento de Electrónica y Electromagnetismo, Facultad de Física, \\ Universidad de Sevilla, Avda. Reina Mercedes s/n, 41012 Sevilla, Spain
}

(Received 12 November 2013; accepted 26 February 2014; published online 19 March 2014)

\begin{abstract}
This paper presents the linear stability analysis of an interface between air and an insulating liquid subjected to a perpendicular electric field, in the presence of unipolar injection of charge. Depending on the characteristics of the liquid and the depth of the liquid layer two different instability thresholds may be found. One of them is characterized by a wavelength of the order of the liquid layer thickness and corresponds to the well-known volume instability of a liquid layer subjected to charge injection. The other one is characterized by a wavelength some ten times the liquid layer thickness and corresponds to the so-called rose-window instability, an instability associated to the balance of surface stresses. @ 2014 AIP Publishing LLC. [http://dx.doi.org/10.1063/1.4868365]
\end{abstract}

\section{INTRODUCTION}

Surface Electrohydrodynamic (EHD) instability of fluid layers under an electric field perpendicular to the interface has been extensively studied in the case of no charge injection. Taylor and McEwan, ${ }^{1}$ in a classical work, described theoretical and experimentally the instability of a layer of perfect conducting liquid placed between two plane electrodes. Above certain electric field, of the order of some $\mathrm{kV} / \mathrm{cm}$, the liquid surface becomes unstable.

Melcher $^{2}$ generalized the study to the case of an interface between two liquids of different conductivities and permittivities. The various possibilities are summarized in the book by Melcher. ${ }^{2}$ The works by Mima et al. ${ }^{3}$ and Giannetta and Ikezi ${ }^{4}$ studied the dynamics of a helium liquid surface negatively charged. They considered that the charge is bounded to the interface, neglecting any mechanism of charge transport in the liquid bulk.

On the other hand, EHD instabilities may be induced in the liquid bulk by the existence of a space charge. This is the case when the electric relaxation time of the liquid $\epsilon / \sigma$ is greater than the time of transit of ions through the liquid layer, $D /\left(K_{l} E\right)(\epsilon$ is the liquid permittivity, $\sigma$ the liquid conductivity, $K_{l}$ the ion mobility in the liquid, $D$ the liquid layer thickness, and $E$ the average electric field in the liquid). In these circumstances, an ion can traverse the liquid layer before being neutralized, there is a net electric charge in the bulk and the electric conduction regime is not Ohmic. The kind of instability that appears in such a highly insulating liquid is referred to as the instability induced by unipolar injection.

Liquid instability induced by unipolar injection is a classical EHD instability, and it has been the subject of many experimental as well as theoretical studies. ${ }^{5-8}$ It is characterized by the emergence of a hexagonal pattern of cells with a typical size of the order of the liquid layer thickness. The liquid motion starts above a critical value of the potential difference in the liquid layer.

Atten and Moreau ${ }^{5}$ conducted an extensive mathematical analysis of the linear stability of a liquid layer subjected to unipolar injection of charge. They considered a liquid layer confined

\footnotetext{
a)rchicon@um.es

b)alberto@us.es
} 
between two plane electrodes and computed the instability threshold for different levels of injection. However, in the case of non-rigid electrodes they did not take into account the deformation of the liquid surface, where gravity and surface tension effects may play an important role.

Watson et al. ${ }^{6}$ studied experimentally the EHD unipolar injection instability using an electron beam to inject charge into the liquid. The liquid surface was free, but they did not consider the interfacial stresses in their theoretical analysis, ${ }^{7}$ which is equivalent to one of the cases studied by Atten and Moreau. ${ }^{5}$

Several devices can be envisaged to inject charge in the liquid, one of them being corona discharge. Corona discharge in air, for example, in a point-to-plate geometry, can induce instabilities on a liquid-air interface. Some descriptive works on the EHD instabilities due to unipolar charge injection using corona discharge were made by Herrick, ${ }^{9}$ Ahmed El-Haddad et al.,${ }^{10}$ and Malraison and Atten. ${ }^{11}$

The electric forces, acting on the surface or in the liquid volume, are responsible for these instabilities. Whether the dominant driving forces are surface or volume forces depends on the liquid conductivity, specifically on the ratio between the charge relaxation time and the time of flight of ions referred to above.

Rose-window instability appears when a corona discharge is applied on a liquid surface, and was described by one of the authors. ${ }^{12,13}$ The instability appears for liquids of low enough electrical conductivity, and it is related to the fact that the electric field pushes the charged liquid surface. The fingerprint of this instability is that, just above the onset, the size of the cells is much larger than the liquid layer thickness.

A more detailed experimental research was reported in a later paper. ${ }^{14}$ For very high insulating liquids, like silicone oil, two convective patterns may coexist. One pattern features small cells with a typical size of the order of the liquid layer thickness. The appearance of this pattern is associated with a certain value of the product $I D^{3}$, which is the relevant parameter in the experiment, where $I$ is the corona electric current. Since the corona current determines the voltage drop through the liquid layer thickness, this instability corresponds to the instability induced by unipolar injection. A second pattern is also observed in silicone oil: the above referred rose-window.

For less insulating liquids than silicone oil, like castor oil or corn oil, the small cell pattern is not visible. Only the rose-window is observed in these liquids. It is important to note that the electric conductivity is in the range of $10^{-11} \mathrm{~S} / \mathrm{m}$ for castor or corn oil compared to $10^{-13} \mathrm{~S} / \mathrm{m}$ for silicone oil.

Besides the size of the cells, other characteristics distinguish experimentally the rose-window instability from the EHD volume instability. In particular, the size of the cells neatly decreases when the electric field increases, whereas in the EHD volume instability this size remains approximately unchanged for a wide range of the applied voltage.

Rose-window instability sets in above a certain critical voltage. For highly insulating liquids, like silicone oil, the critical voltage increases as the liquid layer thickness decreases, becoming very high for very thin layers. On the contrary, for castor or corn oil the critical voltage decreases slowly as the thickness of the liquid layer decreases.

Actually it is not the conductivity of the liquid alone which determines what patterns is observed, but the ratio $S=D^{2} \sigma / \epsilon K_{l} V$. If $S \ll 1$, the charge relaxation time is greater than the ionic transit time, and the ions can traverse the liquid layer without being discharged. There exists a volume charge and the instability due to volume forces can develop. If $S \gg 1$, there cannot exist any volume charge, and the only instability mechanism is the one associated to surface electrical forces (Table I).

There are some previous theoretical works on the surface instability of a liquid-air interface subjected to injection from the air. Atten and Koulova-Nenova ${ }^{15}$ studied the hydrostatic solution and calculated the electric pressure on the liquid layer for the very insulating liquid case. They found that the electric pressure as a function of the liquid layer thickness $D$ has a maximum. Their argument is that the flat surface is potentially unstable only when the electric pressure is a decreasing function of the layer thickness. This would imply that the instability critical voltage becomes very high for very thin layers, consistently with the experimental findings.

In a subsequent work, Koulova-Nenova and Atten ${ }^{16}$ solved numerically the equations for the linear instability of a layer of very insulating liquid. Their physical model differs from that by Atten 
TABLE I. Typical values of the physical properties of some relevant liquids, $\varepsilon$ is the relative electric permittivity and $v$ is the kinematic viscosity.

\begin{tabular}{|c|c|c|c|c|c|c|c|}
\hline & $\varepsilon$ & $\rho\left(\mathrm{kg} / \mathrm{m}^{3}\right)$ & $v\left(\mathrm{~m}^{2} / \mathrm{s}\right)$ & $K\left(\mathrm{~m}^{2} / \mathrm{V} \mathrm{s}\right)$ & $\sigma\left(\Omega^{-1} \mathrm{~m}^{-1}\right)$ & $\begin{array}{c}\text { Relaxation } \\
\text { time (s) }\end{array}$ & $\begin{array}{c}\text { Ion transit } \\
\text { time }(\mathrm{s})(D=1 \mathrm{~mm}, \\
V=100 \mathrm{~V})\end{array}$ \\
\hline $\operatorname{Air}(300 \mathrm{~K})$ & 1.0 & 1.164 & $1.57 \times 10^{-5}$ & $\sim 1 \times 10^{-4}$ & $\sim 1 \times 10^{-15}$ & $9 \times 10^{3}$ & $1 \times 10^{-4}$ \\
\hline Silicone oil & 2.73 & 960 & $50 \times 10^{-6}$ & $5 \times 10^{-10}$ & $6 \times 10^{-13}$ & 40 & 20 \\
\hline Corn oil & 3.1 & 990 & $55 \times 10^{-6}$ & $4.5 \times 10^{-10}$ & $1.9 \times 10^{-11}$ & 1.44 & 22 \\
\hline Castor oil & 4.69 & 958 & $600 \times 10^{-6}$ & $4 \times 10^{-11}$ & $7 \times 10^{-11}$ & 0.6 & 250 \\
\hline Water $(300 \mathrm{~K})$ & 76 & 998 & $7.99 \times 10^{-7}$ & $\sim 5.19 \times 10^{-8}\left(\mathrm{Na}^{+}\right)$ & $\sim 5.5 \times 10^{-6}$ & $1 \times 10^{-4}$ & 1 \\
\hline
\end{tabular}

and Moreau, ${ }^{5}$ or Schneider and Watson, ${ }^{7}$ in that it includes surface tension, gravity, and electrical stresses on the surface. Also, the injection is produced from the air. However, their numerical results amount to just a small correction of the instability threshold found in the previous studies of the instability induced by unipolar injection. No indication of anything similar to rose-window instability was found. Later, they presented some partial results that might indicate the presence of rose-window instability, ${ }^{17}$ but a systematic analysis was not pursued. Also in these works a simplified model for the rose-window instability is presented. This model is based on the assumption that this instability appears at very large wavelengths. In this limit, the authors computed the change of the electric pressure due to a small perturbation of the surface. From the balance between the electric and gravity forces, they obtained a value of the critical voltage as a function of the liquid layer thickness. The most conspicuous feature of the instability threshold so obtained is the existence of a limit liquid layer thickness below which the instability is no longer possible. As it is shown below, neither the assumptions nor the predictions of this simplified model are sustained by the numerical results.

Vega and Pérez ${ }^{18}$ studied the linear EHD instability of an air-liquid interface subjected to an injection of charge. The liquid was modeled as an Ohmic one, and the liquid velocity was neglected under the assumption that the electric pressure on the surface was responsible for the instability mechanism. A first important observation was that the sign of the surface charge depends on the parameter $S$. For small $S$, the charge on the liquid surface is of the same sign than the injected charge, whereas for large $S$ it is the opposite. For very large $S$, the liquid acts like an electrode and the problem becomes similar to that studied by Taylor and McEwan. ${ }^{1}$ The wavenumber of the most unstable mode was found to be zero, indicating very large convective cells. There were also a range of values of $S$ where instability did not exist, corresponding to an almost discharged interface. Although interface tension was taken into account in this study, the fact that the wavenumber of the most unstable mode is zero makes its role negligible, and therefore, changing the surface tension coefficient, or, correspondingly, the Bond number, does not change the critical value. What mainly determines the instability threshold is the interplay between the electric pressure and gravity.

Chicón and Pérez ${ }^{19}$ extended the previous work by considering the role of the liquid velocity. The main finding of that paper was that the inclusion of the liquid velocity shifts the wavenumber of the most unstable mode from zero to a finite value. At the instability threshold, the size of the convective cells is large compared to the liquid layer thickness, as it is found experimentally for rose-window instability. The critical voltage was found to be an increasing function of the liquid layer thickness, in agreement with the experiments for moderately insulating liquids. The range of values of $S$ where instability is forbidden was also found. The critical voltage was studied as a function of the liquid conductivity (Figure 4 in Ref. 19). For conductivities below $10^{-10} \mathrm{~S} / \mathrm{m}$, and liquid layer thickness of the order of $1 \mathrm{~mm}$, the critical voltage is below $3 \mathrm{kV}$. Then the critical voltage increases very fast as the conductivity increases. This is due to the fact that the surface is less charged when the conductivity increases. Then, around $\sigma=10^{-7} \mathrm{~S} / \mathrm{m}$ there is a region where very high voltages, not attainable experimentally, are required for instability. For these conductivities, the surface is almost discharged. Finally, for conductivities greater than $\sigma=10^{-6} \mathrm{~S} / \mathrm{m}$ the critical voltage is of the order of some $15 \mathrm{kV}$. In this regime, the surface is charged with a charge opposite 
to that of the injecting electrode, the liquid acts as an electrode, and the instability is again the one studied by Taylor and McEwan. ${ }^{1}$

Since experiments show that rose-window instability is also observed in very insulating liquids, it was a most natural step to analyze the same theoretical problem for a non-Ohmic model. In this paper, the linear stability of a non-Ohmic liquid-air interface subjected to an injection of ions from the air is studied in detail. At first, for a typical layer thickness above $1 \mathrm{~mm}$, no sign of an instability with convective cells much larger than the liquid thickness was found. The stability curves, which give the critical voltage as a function of the wavenumber of the perturbation, show only the ever-present minimum associated to the volume instability, located at a wavenumber around $k=4$.

A few years ago, working on Bénard convection, Schatz ${ }^{20}$ et al. and VanHook et al. ${ }^{21}$ showed that a long-wavelength instability supplants hexagonal convection cells in very thin liquid layers. Bénard convection and EHD instabilities of a dielectric liquid layer are different problems, but they have in common that surface tension and gravity are relevant terms in the stress balance at the surface. And it is the balance of the different driving and restoring terms which determines the wavenumber selection in several instability problems. This suggests the idea of studying the effect of significantly decreasing the liquid layer thickness. And it is under these conditions that a new minimum is found for very insulating liquids.

In Sec. II, the basic equations are written, the unperturbed solution obtained, linear perturbations added, and normal mode analysis is carried out.

The most important feature of the non-Ohmic model that is not taken into account in the Ohmic analysis is the possible appearance of volume charge, which has its own instability mechanism. The surface charge that is attributed to the surface of an Ohmic conductor is a limit case of a volume charge extended over a very thin liquid depth. In order to study this transition between volume and surface charge, a mixed model is introduced. Section III is devoted to the analysis of this model, where the residual conductivity of the liquid is taken into account. Finally, Sec. IV presents the numerical method employed to solve the equations and the results obtained.

\section{POSITION OF THE PROBLEM}

\section{A. Basic equations}

The configuration to be studied is that of two horizontal electrodes kept a distance $h$ apart, the lower one grounded and the upper one at potential $V$. The space between the electrodes is filled with an insulating liquid layer disposed on the lower electrode and an air gap (Fig. 1). The thickness of the liquid layer is $D$ and that of the air gap $L=h-D$. Charge is injected from the upper electrode, and a space charge distribution builds up in the air gap and in the liquid bulk. The relevant properties of the liquid are its permittivity $\epsilon_{l}$, density $\rho$, viscosity $\eta$, and surface tension $\gamma$. A system of coordinates is chosen such that the $z$-axis is vertical, with the unit vector $\mathbf{e}_{z}$ pointing downwards. The unperturbed liquid surface is located at $z=0$, the upper electrode at $z=-L$, and the lower one at $z=D$.

The space charge distribution in the air is governed by the equations

$$
\begin{gathered}
\nabla^{2} \phi=-\frac{q}{\epsilon_{0}}, \\
\frac{\partial q}{\partial t}+\nabla \cdot \mathbf{J}=0,
\end{gathered}
$$

with $\mathbf{J}=K_{a} q \mathbf{E}$. Here, $q$ means charge density, $K_{a}$ ion mobility, $\mathbf{E}$ electric field, and $\phi$ electric potential. An average mobility $K_{a}$ is assumed for the ions. As the drift velocity of ions in air is usually much greater than the fluid velocity, a convection term $(q \mathbf{u})$ can be neglected in the expression for the current density $\mathbf{J}$. A term that would correspond to charge diffusion is also neglected, as it is usual in this type of problems. ${ }^{8}$

It is assumed that the mechanical equation in the air gap is that of hydrostatic equilibrium

$$
-\nabla p+\rho_{a} g \mathbf{e}_{z}+q \mathbf{E}=0,
$$

where $p$ is the pressure, $\rho_{a}$ the air mass density, and $g$ the acceleration of gravity. 


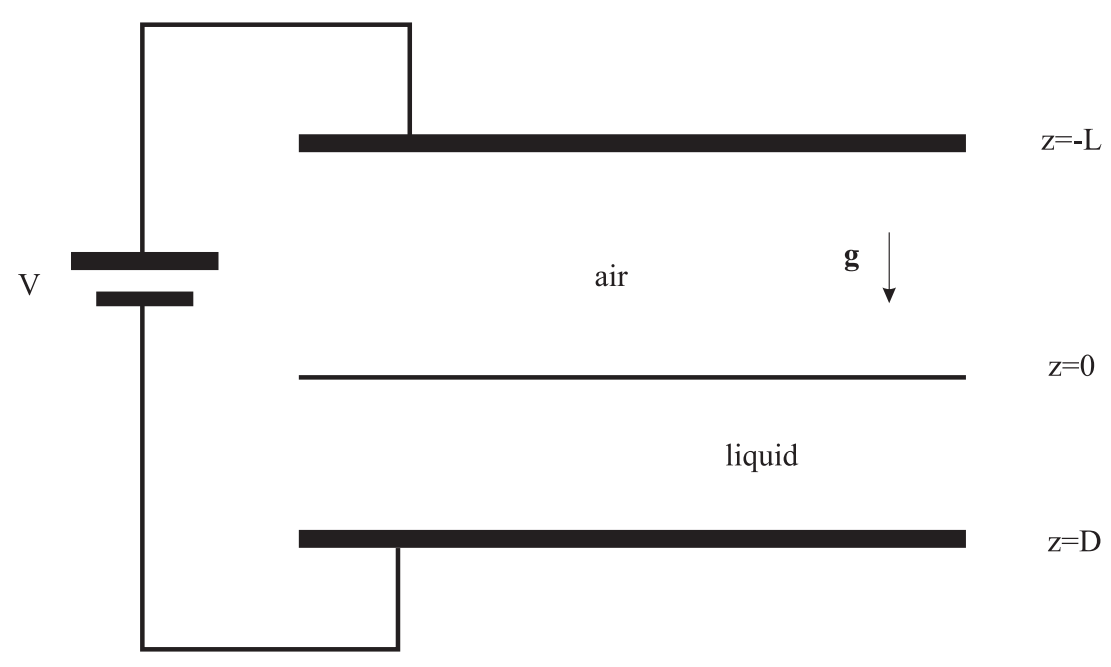

FIG. 1. Geometrical configuration of the system.

This equation is only an approximation when the liquid surface is perturbed. This is so because any wavy perturbation of the liquid surface will produce a gradient of the charge density with a non-zero horizontal component. Then, the last term in the left-hand side of the equation will have a curl $\nabla q \times \mathbf{E}$ different from zero, so that it cannot be balanced by any pressure term. However, due to the large difference in the values of viscosity for both fluids, the influence of air movement above the interface upon the liquid below would be small, and the problem can be formulated in a first approximation without solving for the velocity field in the air.

The equations governing the space charge distribution in the liquid bulk are

$$
\begin{gathered}
\nabla^{2} \phi=-\frac{q}{\epsilon_{l}}, \\
\frac{\partial q}{\partial t}+\nabla \cdot \mathbf{J}=0,
\end{gathered}
$$

with $\mathbf{J}=q\left(K_{l} \mathbf{E}+\mathbf{u}\right)$. Here, $K_{l}$ denotes the mobility of ions in the liquid and $\mathbf{u}$ the liquid velocity.

Finally, the equations that govern the motion of the liquid are

$$
\begin{gathered}
\nabla \cdot \mathbf{u}=0 \\
\rho\left(\frac{\partial \mathbf{u}}{\partial t}+\mathbf{u} \cdot \nabla \mathbf{u}\right)=-\nabla p+\rho g \mathbf{e}_{z}+\eta \nabla^{2} \mathbf{u}+q \mathbf{E} .
\end{gathered}
$$

The main difference, from a mathematical point of view, between the problem analyzed in this paper for an insulating liquid and the analogous problem for an Ohmic liquid, ${ }^{19}$ is that the existence of a space charge density $q$ inside the liquid couples the electrical and mechanical equations, as it is evident from Eqs. (5) and (7).

A number of boundary conditions are to be added to the system of partial differential equations above. At the electrodes, which are rigid in the experimental setup, these are

$$
\begin{gathered}
\phi=V, \quad q=q_{0} \quad \text { at } \quad z=-L, \\
\phi=0, \quad \mathbf{u}=0 \quad \text { at } \quad z=D .
\end{gathered}
$$

It is assumed in Eq. (8) that the charge density at the injecting electrode remains constant, and independent of any changes in the electric field. This is known as the autonomous injection hypothesis. It has been discussed at length elsewhere ${ }^{8}$ that the hypothesis is fully justified when the injection strength is large enough, the so called space-charge-limited-current (SCLC) regime. This is the regime often met in experiments. 
The remaining boundary conditions correspond to the liquid-air interface. Let $[A]=A_{l}-A_{a}$ denote the jump of any quantity $A$ across the interface.

The discontinuity of the electric field across the interface is ruled by the equations

$$
\begin{aligned}
& {[\mathbf{E}] \times \mathbf{n}=0,} \\
& {[\epsilon \mathbf{E}] \cdot \mathbf{n}=0,}
\end{aligned}
$$

where $\epsilon$ denotes the permittivity of the corresponding medium, and $\mathbf{n}$ the unit vector normal to the surface and pointing towards the liquid. The right-hand side of Eq. (10) is zero due to the absence of a surface charge for insulating liquids. This in fact simplifies the equation corresponding to conservation of charge across the interface, ${ }^{22}$ which now takes the form

$$
[\mathbf{J}] \cdot \mathbf{n}-[q \mathbf{u}] \cdot \mathbf{n}=0 .
$$

The fact that the liquid surface is a material one is expressed by the kinematic equation

$$
-\frac{\partial f}{\partial t}+u_{z}-u_{x} \frac{\partial f}{\partial x}-u_{y} \frac{\partial f}{\partial y}=0,
$$

where the interface is defined by a function $z=f(x, y, t)$.

Finally, the balance of tangential and normal total stresses at the interface is given by

$$
\begin{gathered}
\eta \mathbf{t} \cdot\left(\nabla \mathbf{u}+\nabla \mathbf{u}^{T}\right) \cdot \mathbf{n}=0, \\
-[p]+\eta \mathbf{n} \cdot\left(\nabla \mathbf{u}+\nabla \mathbf{u}^{T}\right) \cdot \mathbf{n}+\left[\epsilon(\mathbf{E} \cdot \mathbf{n})^{2}\right]-\left[\frac{1}{2} \epsilon E^{2}\right]-\gamma\left(\nabla_{s} \cdot \mathbf{n}\right)=0,
\end{gathered}
$$

respectively, where $\mathbf{t}$ is any unit vector tangent to the surface and $\nabla_{s}$ is the surface gradient operator. $^{22,23}$

The last equation plays a key role in the problem. Some essential features of the rose-window instability are consequences of the balance of normal stresses.

\section{B. Non-dimensional equations}

The non-dimensional parameters relevant to the problem arise in a natural way when suitable units adapted to the system are chosen. Thus, $D$ is taken as unit of distance, $\rho D^{2} / \eta$ of time, $\eta^{2} /\left(\rho D^{2}\right)$ of pressure, $V / D$ of electric field, $\epsilon_{l} V / D^{2}$ of charge, and $\epsilon_{l} K_{l} V^{2} / D^{3}$ of electric current density. If the same symbols are used for the non-dimensional quantities as for their dimensional counterparts, the non-dimensional equations read as follows.

In the air,

$$
\begin{gathered}
\nabla^{2} \phi=-\epsilon_{r} q, \\
\frac{M}{U^{1 / 2}} \frac{\partial q}{\partial t}+\nabla \cdot \mathbf{J}=0, \quad \text { with } \quad \mathbf{J}=\frac{K_{a}}{K_{l}} q \mathbf{E}, \\
-\nabla p+\frac{\rho_{a}}{\rho} g^{*} \mathbf{e}_{z}+U q \mathbf{E}=0 .
\end{gathered}
$$

In the liquid,

$$
\begin{gathered}
\nabla^{2} \phi=-q, \\
\frac{M}{U^{1 / 2}} \frac{\partial q}{\partial t}+\nabla \cdot \mathbf{J}=0, \quad \text { with } \quad \mathbf{J}=q\left(\mathbf{E}+\frac{M}{U^{1 / 2}} \mathbf{u}\right), \\
\nabla \cdot \mathbf{u}=0, \\
\frac{\partial \mathbf{u}}{\partial t}+\mathbf{u} \cdot \nabla \mathbf{u}=-\nabla p+g^{*} \mathbf{e}_{z}+\nabla^{2} \mathbf{u}+U q \mathbf{E} .
\end{gathered}
$$


The seemingly strange factor $M / U^{1 / 2}$ in the equations for charge conservation comes from the fact that the unit of electric current density has been defined in terms of the drift velocity of the ions in the liquid, instead of the velocity of the liquid.

The boundary conditions at the electrodes are

$$
\begin{gathered}
\phi=1, \quad q=C \text { at } z=-L, \\
\phi=0, \quad \mathbf{u}=0 \quad \text { at } z=1,
\end{gathered}
$$

and the boundary conditions at the interface are

$$
\begin{gathered}
{[\mathbf{E}] \times \mathbf{n}=0,} \\
{[\epsilon \mathbf{E}] \cdot \mathbf{n}=0,} \\
{[\mathbf{J}] \cdot \mathbf{n}-\frac{M}{U^{1 / 2}}[q \mathbf{u}] \cdot \mathbf{n}=0,} \\
-\frac{\partial f}{\partial t}+u_{z}-u_{x} \frac{\partial f}{\partial x}-u_{y} \frac{\partial f}{\partial y}=0, \\
\mathbf{t} \cdot\left(\nabla \mathbf{u}+\nabla \mathbf{u}^{T}\right) \cdot \mathbf{n}=0, \\
-[p]+\mathbf{n} \cdot\left(\nabla \mathbf{u}+\nabla \mathbf{u}^{T}\right) \cdot \mathbf{n}+\frac{U}{\epsilon_{r}}\left(\left[\frac{\epsilon}{\epsilon_{0}}(\mathbf{E} \cdot \mathbf{n})^{2}\right]-\left[\frac{1}{2} \frac{\epsilon}{\epsilon_{0}} E^{2}\right]\right)-\frac{g^{*}}{B o}\left(\nabla_{s} \cdot \mathbf{n}\right)=0 .
\end{gathered}
$$

The nondimensional parameters relevant to the problem are

$$
\begin{array}{rlrl}
U=\frac{\epsilon_{l} \rho V^{2}}{\eta^{2}}, & M=\frac{1}{K_{l}} \sqrt{\frac{\epsilon_{l}}{\rho}}, & C=\frac{q_{0} D^{2}}{\epsilon_{l} V}, \\
g^{*}=\frac{\rho^{2} D^{3}}{\eta^{2}} g, & \text { Вo }=\frac{\rho g D^{2}}{\gamma} .
\end{array}
$$

The parameter $U$ represents the ratio of the destabilizing electric force to the stabilizing viscous force, $M$ is the ratio of the so-called hydrodynamic mobility to the ionic mobility in the liquid, and $C$ is a measure of the injection strength. Finally, $g^{*}$ is a nondimensional measure of the acceleration of gravity, $\epsilon_{r}$ is the relative permittivity of the liquid, and Bo is the usual Bond number, representing the ratio between the gravity and capillary forces.

\section{Static solution}

The equations admit a steady hydrostatic solution with a flat interface. The electric field, charge density, and pressure in the air are given by

$$
\begin{gathered}
E_{0 z}(z)=a(z+b)^{1 / 2}, \\
Q_{0}(z)=\frac{a /\left(2 \epsilon_{r}\right)}{(z+b)^{1 / 2},} \\
p_{0}(z)=P_{0 a}+\left(\frac{\rho_{a}}{\rho} g^{*}+U J_{0} \frac{K_{l}}{K_{a}}\right)(z+L),
\end{gathered}
$$

and in the liquid by

$$
\begin{gathered}
E_{0 z}(z)=c(z+d)^{1 / 2}, \\
Q_{0}=\frac{c / 2}{(z+d)^{1 / 2}}, \\
p_{0}(z)=P_{0 l}+\left(g^{*}+U J_{0}\right) z,
\end{gathered}
$$


where $J_{0}$ is the uniform current density. The constants $a, b, c$, and $d$ appearing in the solution are functions of the nondimensional parameters, and are obtained from the boundary conditions as the solution of the algebraic equations

$$
\begin{aligned}
\frac{4}{3} \epsilon_{r} C(b-L)^{1 / 2}\left[b^{3 / 2}-(b-L)^{3 / 2}\right. & \left.+\sqrt{\frac{K_{a}}{\epsilon_{r} K_{l}}}\left(\left(1+\frac{K_{l}}{\epsilon_{r} K_{a}} b\right)^{3 / 2}-\left(\frac{K_{l}}{\epsilon_{r} K_{a}} b\right)^{3 / 2}\right)\right]=1, \\
a & =2 \epsilon_{r} C(b-L)^{1 / 2} \\
c & =a \sqrt{\frac{K_{a}}{\epsilon_{r} K_{l}}} \\
d & =b \frac{K_{l}}{\epsilon_{r} K_{a}} .
\end{aligned}
$$

The values of the mechanical pressure at both sides of the interface are related through Eq. (28), and they ultimately depend on the a priori given value $P_{0 a}$ of air pressure near the injecting electrode.

It is well known that a space charge in the bulk of the liquid which decreases with the distance from the injecting electrode is a potentially unstable configuration. This is the origin of the well studied classical EHD instability, with convective hexagonal cells with a size of the order of the liquid layer thickness. ${ }^{5}$

The possibility of deformation of the initially flat free interface between liquid and air is another source of potential instability. In the Ohmic case, Vega and Pérez ${ }^{18}$ showed that the electric pressure is a decreasing function of the liquid layer thickness. This is a potentially unstable situation, since any fluctuation leading to the decrease of the layer thickness would be amplified. Although in the non-Ohmic case there is not a surface charge, the volume space charge is greater near the surface, and can be imagined as an effective surface charge. Therefore, a similar instability mechanism may be expected.

\section{Linear stability}

The mathematical treatment of the instability of the steady state is carried out following a well-known procedure. ${ }^{24}$ First, it is assumed that the physical quantities which define the state of the system suffer small perturbations, and the equations governing the perturbations are obtained. Then, all terms of order higher than the first in the perturbations are neglected, arriving at a set of linear equations. Specifically, any variable $\psi(x, y, z, t)$ in the system is written as $\psi=\Psi_{0}(z)+\psi^{\prime}(x, y, z$, $t$ ), where $\Psi_{0}$ corresponds to its value in the steady state and $\psi^{\prime}$ to the perturbation. After substitution into the general equations and linearization, the equations governing the perturbations in the air are

$$
\begin{gathered}
\nabla^{2} \phi^{\prime}=-\epsilon_{r} q^{\prime}, \\
\frac{K_{l}}{K_{a}} \frac{M}{U^{1 / 2}} \frac{\partial q^{\prime}}{\partial t}+\frac{d Q_{0}}{d z} E_{z}^{\prime}+2 \epsilon_{r} Q_{0} q^{\prime}+E_{0 z} \frac{\partial q^{\prime}}{\partial z}=0, \\
-\nabla p^{\prime}+U\left(Q_{0} \mathbf{E}^{\prime}+q^{\prime} \mathbf{E}_{0}\right)=0,
\end{gathered}
$$

and in the liquid

$$
\begin{gathered}
\nabla^{2} \phi^{\prime}=-q^{\prime}, \\
\frac{M}{U^{1 / 2}} \frac{\partial q^{\prime}}{\partial t}+\frac{d Q_{0}}{d z} E_{z}^{\prime}+2 Q_{0} q^{\prime}+E_{0 z} \frac{\partial q^{\prime}}{\partial z}+\frac{M}{U^{1 / 2}} \frac{d Q_{0}}{d z} u_{z}=0, \\
\nabla \cdot \mathbf{u}=0,
\end{gathered}
$$




$$
\frac{\partial \mathbf{u}}{\partial t}=-\nabla p^{\prime}+\nabla^{2} \mathbf{u}+U\left(Q_{0} \mathbf{E}^{\prime}+q^{\prime} \mathbf{E}_{0}\right)
$$

The mechanical pressure $p^{\prime}$ can be eliminated in Eq. (44) by taking the curl on both sides. In fact, it is convenient to apply the curl operator twice, for this permits to take full advantage of Eq. (43) and leads to three independent equations for the components of the liquid velocity $\mathbf{u}$, namely,

$$
\left(\nabla^{2}-\frac{\partial}{\partial t}\right) \nabla^{2} \mathbf{u}=U \nabla \times \nabla \times\left(Q_{0} \mathbf{E}^{\prime}+q^{\prime} \mathbf{E}_{0}\right) .
$$

Only the z-component of Eq. (45) is needed to solve the stability problem. This is so because $u_{z}$ is the only component of the liquid velocity $\mathbf{u}$ appearing in the equation corresponding to charge conservation in the liquid, Eq. (42), and the same is true for the boundary conditions at the interface and at the lower electrode.

The boundary equations for the perturbations at the electrodes are simple

$$
\begin{aligned}
\phi^{\prime}=0, & q^{\prime}=0 \quad \text { at } z=-L, \\
\phi^{\prime}=0, & \mathbf{u}=0 \text { at } z=1 .
\end{aligned}
$$

However, the treatment of boundary conditions at the perturbed interface requires special attention. They involve terms of the form $[\psi]_{z_{s}}=\psi_{l}\left(x, y, z_{s}, t\right)-\psi_{a}\left(x, y, z_{s}, t\right)$, with $z_{s}=f(x$, $y, t)$ and $\psi$ some physical quantity. The equations are complicated by the fact that the shape $z_{s}$ of the interface is not known in advance. A procedure to overcome this difficulty can be outlined as follows. ${ }^{25}$ Given a function $\psi(x, y, z, t)$ whose domain is the liquid bulk or the air gap, the limit value at the perturbed interface can be written as

$$
\psi\left(x, y, z_{s}, t\right)=\psi(x, y, z=0, t)+\left(\frac{\partial \psi}{\partial z}\right)_{z=0} z_{s}+\cdots .
$$

Putting $\psi=\Psi_{0}+\psi^{\prime}$ and retaining terms up to the first order leads to

$$
\psi\left(x, y, z_{s}, t\right)=\Psi_{0}(z=0)+\psi^{\prime}(x, y, z=0, t)+\left(\frac{d \Psi_{0}}{d z}\right)_{z=0} z_{s}+\cdots .
$$

By doing this on both sides of the interface, the jump $[\psi]_{z_{s}}$ across the perturbed interface in the linear approximation can be expressed in terms of jumps across the unperturbed interface $z=0$

$$
[\psi]_{z_{s}}=\left[\Psi_{0}\right]_{z=0}+\left[\psi^{\prime}\right]_{z=0}+\left[\frac{d \Psi_{0}}{d z}\right]_{z=0} f(x, y, t) .
$$

Therefore, the boundary conditions for the perturbations at the interface become

$$
\begin{gathered}
{\left[\phi^{\prime}\right]_{0}=-\left[\frac{d \Phi_{0}}{d z}\right]_{0} f=\left[E_{0 z}\right]_{0} f,} \\
{\left[\frac{\epsilon}{\epsilon_{0}} \frac{\partial \phi^{\prime}}{\partial z}\right]_{0}=\left[\frac{\epsilon}{\epsilon_{0}} \frac{d E_{0 z}}{d z}\right]_{0} f,} \\
{\left[\frac{K}{K_{l}}\left(\frac{\epsilon}{\epsilon_{l}} E_{0 z} \nabla^{2} \phi^{\prime}+Q_{0} \frac{\partial \phi^{\prime}}{\partial z}\right)\right]_{0}=0,} \\
-\frac{\partial f}{\partial t}+u_{z}(0+)=0, \\
\nabla_{s}^{2} u_{z}(0+)-\frac{\partial^{2} u_{z}}{\partial z^{2}}(0+)=0, \\
-\left[p^{\prime}\right]_{0}-\left(1-\frac{\rho_{a}}{\rho}\right) g^{*} f+2 \frac{\partial u_{z}}{\partial z}(0+)-\frac{U}{\epsilon_{r}}\left[\frac{\epsilon}{\epsilon_{0}} E_{0 z} \frac{\partial \phi^{\prime}}{\partial z}\right]_{0}+\frac{g^{*}}{\mathrm{Bo}} \nabla_{s}^{2} f=0 .
\end{gathered}
$$


Wherever $K$ and $\epsilon$ appear, they refer to the ionic mobility and permittivity of the corresponding medium, respectively. Equation (51) represents the equilibrium of tangential stresses. It is derived from Eq. (27) by considering two mutually perpendicular unit vectors $\mathbf{t}$ on the tangent plane to the interface, combining the results and taking into account the incompressibility of the liquid.

The pressure term $\left[p^{\prime}\right]_{0}$ in Eq. (52) can be eliminated, as it was previously done in the differential equations. In the air gap, differentiating the $x$ - and $y$-components of Eq. (40) with respect to $x$ and $y$, respectively, leads to

$$
-\nabla_{s}^{2} p^{\prime}=U Q_{0} \nabla_{s}^{2} \phi^{\prime}
$$

Proceeding analogously and taking into account the incompressibility of the liquid, Eq. (44) gives

$$
-\nabla_{s}^{2} p^{\prime}=\frac{\partial^{3} u_{z}}{\partial z^{3}}+\nabla_{s}^{2} \frac{\partial u_{z}}{\partial z}-\frac{\partial}{\partial t} \frac{\partial u_{z}}{\partial z}+U Q_{0} \nabla_{s}^{2} \phi^{\prime}
$$

Then, the jump $\left[\nabla_{s}^{2} p^{\prime}\right]_{0}$ is obtained by taking the limit as $z \rightarrow 0+$ in Eq. (54), $z \rightarrow 0-$ in Eq. (53) and subtracting. Upon applying the operator $\nabla_{s}^{2}$ to Eq. (52) and substituting $\left[\nabla_{s}^{2} p^{\prime}\right]_{0}$ into the ensuing equation, the equilibrium of normal stresses at the interface is finally expressed as

$$
\begin{aligned}
\left(3 \nabla_{s}^{2}-\frac{\partial}{\partial t}\right) \frac{\partial u_{z}}{\partial z}(0+)+\frac{\partial^{3} u_{z}}{\partial z^{3}}(0+)+ & U\left(\left[Q_{0} \nabla_{s}^{2} \phi^{\prime}\right]_{0}-\frac{1}{\epsilon_{r}}\left[\frac{\epsilon}{\epsilon_{0}} E_{0 z} \nabla_{s}^{2} \frac{\partial \phi^{\prime}}{\partial z}\right]_{0}\right) \\
& -\left(1-\frac{\rho_{a}}{\rho}\right) g^{\star} \nabla_{s}^{2} f+\frac{g^{\star}}{\mathrm{Bo}_{\mathrm{o}}}\left(\nabla_{s}^{2}\right)^{2} f=0 .
\end{aligned}
$$

\section{E. Normal mode analysis}

Since the perturbation equations are linear, an arbitrary disturbance can be expanded in terms of a suitable complete set of normal modes and the stability of each mode examined individually. The planar geometry of the system, the fact that all physical quantities in the stationary state are functions only of the $z$-coordinate, and Fourier analysis suggest a set of normal modes where the perturbations have the form

$$
\begin{aligned}
f(x, y, t) & =f_{0} e^{\omega t} e^{i\left(k_{x} x+k_{y} y\right)}, \\
\phi^{\prime}(x, y, z, t) & =g(z) e^{\omega t} e^{i\left(k_{x} x+k_{y} y\right)}, \\
u_{z}(x, y, z, t) & =u(z) e^{\omega t} e^{i\left(k_{x} x+k_{y} y\right)},
\end{aligned}
$$

where $\mathbf{k}=\left(k_{x}, k_{y}\right)$ is the two-dimensional wave vector of the disturbance and $\omega$ the growth rate.

The perturbation equations for a given mode are, in the air gap $(-L<z<0)$

$$
\left(\frac{K_{l}}{K_{a}} \frac{1}{\epsilon_{r}} \frac{M}{U^{1 / 2}} \omega+2 Q_{0}+\frac{1}{\epsilon_{r}} E_{0 z} \frac{d}{d z}\right)\left(\frac{d^{2}}{d z^{2}}-k^{2}\right) g+\frac{d Q_{0}}{d z} \frac{d g}{d z}=0,
$$

and in the liquid layer $(0<z<1)$

$$
\begin{gathered}
\left(\frac{M}{U^{1 / 2}} \omega+2 Q_{0}+E_{0 z} \frac{d}{d z}\right)\left(\frac{d^{2}}{d z^{2}}-k^{2}\right) g+\frac{d Q_{0}}{d z} \frac{d g}{d z}-\frac{M}{U^{1 / 2}} \frac{d Q_{0}}{d z} u(z)=0, \\
\left(\frac{d^{2}}{d z^{2}}-k^{2}-\omega\right)\left(\frac{d^{2}}{d z^{2}}-k^{2}\right) u-U k^{2}\left(\frac{d Q_{0}}{d z}-E_{0 z}\left(\frac{d^{2}}{d z^{2}}-k^{2}\right)\right) g=0 .
\end{gathered}
$$

The boundary conditions at the electrodes are simply

$$
\begin{array}{r}
g=0, \quad \frac{d^{2} g}{d z^{2}}=0 \text { at } z=-L, \\
g=0, \quad u=0, \quad \frac{d u}{d z}=0 \text { at } z=1 .
\end{array}
$$


Finally, the boundary conditions at the interface are written as

$$
\begin{gathered}
{[g]_{0}=\left[E_{0 z}\right]_{0} f_{0},} \\
{\left[\frac{\epsilon}{\epsilon_{0}} \frac{d g}{d z}\right]_{0}=\left[\frac{\epsilon}{\epsilon_{0}} \frac{d E_{0 z}}{d z}\right]_{0} f_{0},} \\
{\left[\frac{K}{K_{l}}\left(\frac{\epsilon}{\epsilon_{l}} E_{0 z}\left(\frac{d^{2}}{d z^{2}}-k^{2}\right) g+Q_{0} \frac{d g}{d z}\right)\right]_{0}=0,} \\
-\omega f_{0}+u(0+)=0, \\
\frac{d^{3} u}{d z^{2}}(0+)+k^{2} u(0+)=0, \\
+(0+)-\left(3 k^{2}+\omega\right) \frac{d u}{d z}(0+)+U k^{2}\left(-\left[Q_{0} g\right]_{0}+\frac{1}{\epsilon_{r}}\left[\frac{\epsilon}{\epsilon_{0}} E_{0 z} \frac{d g}{d z}\right]_{0}\right) \\
+k^{2}\left(1-\frac{\rho_{a}}{\rho}+\frac{k^{2}}{\mathrm{Bo}}\right) g^{*} f_{0}=0 .
\end{gathered}
$$

The principle of the exchange of stabilities is assumed to be valid for this problem, so that marginal stability is characterized by $\omega=0 .{ }^{24}$ Thus, as regards the onset of instability, the problem consists in finding the critical value $U(k)$ above which the mode with wavenumber $k$ becomes unstable.

In order to solve the problem numerically, it is convenient that all the equations be defined on a common domain. This can be achieved by expressing the electric potential in the air gap in terms of a new function $h(z)$, defined on the interval $0<z<1$ as $h(z)=g(-L z)$. Thus, the differential equations are finally written as

$$
\begin{gathered}
{\left[\frac{d^{3}}{d z^{3}}-\frac{L}{b-L z} \frac{d^{2}}{d z^{2}}-L^{2}\left(k^{2}+\frac{1}{4} \frac{1}{(b-z L)^{2}}\right) \frac{d}{d z}+L^{3} \frac{k^{2}}{b-L z}\right] h(z)=0,} \\
{\left[\frac{d^{3}}{d z^{3}}+\frac{1}{d+z} \frac{d^{2}}{d z^{2}}-\left(k^{2}+\frac{1}{4} \frac{1}{(d+z)^{2}}\right) \frac{d}{d z}-\frac{k^{2}}{d+z}\right] g(z)+\frac{1}{4} \frac{M}{U^{1 / 2}} u(z)=0,} \\
\left(\frac{d^{2}}{d z^{2}}-k^{2}\right)^{2} u(z)+U k^{2} c\left[(d+z)^{1 / 2}\left(\frac{d^{2}}{d z^{2}}-k^{2}\right)+\frac{1}{4}(d+z)^{-3 / 2}\right] g(z)=0,
\end{gathered}
$$

on the domain $0<z<1$, and the corresponding boundary conditions

$$
\begin{gathered}
h(1)=0, \\
\frac{d^{2} h}{d z^{2}}(1)=0, \\
g(1)=0, \\
u(1)=0, \\
\frac{d u}{d z}(1)=0, \\
g(0)-h(0)=\left(\frac{1}{\epsilon_{r}}-1\right) a b^{1 / 2} f_{0}, \\
\epsilon_{r} \frac{d g}{d z}(0)+\frac{1}{L} \frac{d h}{d z}(0)=\frac{1}{2}\left(\frac{1}{d}-\frac{1}{b}\right) a b^{1 / 2} f_{0},
\end{gathered}
$$




$$
\begin{array}{r}
\frac{d^{2} g}{d z^{2}}(0)+\frac{1}{2 d} \frac{d g}{d z}(0)-k^{2} g(0)-\frac{K_{a}}{K_{l}}\left(\frac{1}{L^{2}} \frac{d^{2} h}{d z^{2}}(0)-\frac{1}{2 b L} \frac{d h}{d z}(0)-k^{2} h(0)\right)=0, \\
u(0)=0, \\
\frac{d^{2} u}{d z^{2}}(0)=0, \\
\frac{d^{3} u}{d z^{3}}(0)-3 k^{2} \frac{d u}{d z}(0)+k^{2} U \frac{a b^{1 / 2}}{\epsilon_{r}}\left(\frac{d g}{d z}(0)+\frac{1}{L} \frac{d h}{d z}(0)-\frac{1}{2}\left(\frac{1}{d} g(0)-\frac{1}{b} h(0)\right)\right) \\
+k^{2}\left(1-\frac{\rho_{a}}{\rho}+\frac{k^{2}}{\mathrm{Bo}}\right) g^{*} f_{0}=0 .
\end{array}
$$

It should be noted that the amplitude $f_{0}$ of the surface disturbance does not enter into the equations as an unknown, but rather plays the role of a normalizing constant, and therefore it can be given any convenient value.

\section{MODEL OF A DIELECTRIC LIQUID WITH RESIDUAL CONDUCTIVITY}

An heuristic way of going from a fully insulating liquid to an Ohmic one is considered in this section. As mentioned before, the Ohmic case was studied in a previous paper ${ }^{19}$ where a surface instability was found. A mixed model is proposed where the current density is assumed to be $\mathbf{J}=$ $q\left(K_{l} \mathbf{E}+\mathbf{u}\right)+\sigma \mathbf{E}$, with $\sigma$ standing for the residual Ohmic conductivity. When units are chosen as in Subsection II B, the nondimensional current density is written as

$$
\mathbf{J}=q\left(\mathbf{E}+\frac{M}{U^{1 / 2}} \mathbf{u}\right)+S \mathbf{E},
$$

where the dimensionless parameter $S=\sigma D^{2} /\left(K_{l} \epsilon_{l} V\right)$ measures the relative strength of Ohmic to non-Ohmic conduction in the liquid. Equations (15)-(21) remain the basic equations of the problem when this new expression is substituted for the current density in the liquid bulk.

The steady hydrostatic solution with a flat air-liquid interface shows charge and electric field distributions in air still given by Eqs. (30)-(32).

However, the solution in the liquid is less simple and Eqs. (33)-(35) do not hold true. Upon integration of the charge conservation equation in the liquid, the electric field is given by the implicit equation

$$
E_{0 z}+\frac{J_{0}}{S} \ln \left(J_{0}-S E_{0 z}\right)=-S z+\alpha,
$$

where $\alpha$ is an integration constant and $J_{0}=\left(K_{a} / K_{l}\right) a^{2} /\left(2 \epsilon_{r}\right)$ represents the uniform electric current density. The three constants $a, b$, and $\alpha$ are to be obtained from the boundary conditions, which in this case are most conveniently expressed in the form

$$
\begin{array}{r}
a=2 \epsilon_{r} C(b-L)^{1 / 2}, \\
\frac{4}{3} \epsilon_{r} C(b-L)^{1 / 2}\left[b^{3 / 2}-(b-L)^{3 / 2}\right]+\frac{J_{0}}{S}-\frac{1}{2 S}\left(E_{0 z}^{2}(1)-E_{0 z}^{2}(0+)\right)=1, \\
E_{0 z}(1)+\frac{J_{0}}{S} \ln \left(J_{0}-S E_{0 z}(1)\right)=-S+E_{0 z}(0+)+\frac{J_{0}}{S} \ln \left(J_{0}-S E_{0 z}(0+)\right),
\end{array}
$$

where $E_{0 z}(0+)$ and $E_{0 z}(1)$ are the values of the electric field at the interface $(z=0)$ and the collecting electrode $(z=1)$, respectively. Taking into account the jump of the electric field across the interface gives $E_{0 z}(0+)=2 C b^{1 / 2}(b-L)^{1 / 2}$. The unknown $E_{0 z}(1)$ can be eliminated from the second equation in Eq. (84), and substitution into the last equation in Eq. (85) finally leads to a single equation in the unknown $b$ which is solved numerically. 
For large values of the parameter $S$, the volume becomes discharged and $J_{0}=S E_{0 z}$, except very close to the interface. The term $\frac{S E_{o z}}{J_{0}}$ can be neglected compared to $\ln \left(1-\frac{S E_{o z}}{J_{0}}\right)$ in Eq. (82), and the electric field is approximated by

$$
E_{0 z}=\frac{J_{0}}{S}(1-B) e^{-S^{2} z / J_{0}},
$$

with $B$ some constant. The corresponding density of electric charge is then

$$
q=S B e^{-S^{2} z / J_{0}} .
$$

Therefore, the charge is essentially confined to a very thin layer of thickness $J_{0} / S^{2}$ close to the interface. In the limit $S \rightarrow \infty$, it becomes a surface charge of value

$$
\sigma_{s}=\int_{0+}^{\infty} q d z=\frac{J_{0}}{S}-E_{0 z}(0+)
$$

Thus, the parameter $S$ serves to control the transition between a regime of a completely insulating liquid $(S=0)$, characterized by a space charge distribution, to a regime corresponding to a very conducting liquid $(S \rightarrow \infty)$, characterized by a surface charge density.

Once the steady solution has been obtained, the analysis of its linear stability proceeds along exactly the same lines as described in Secs. II D and II E for the purely insulating liquid. Only some of the equations have to be modified to include the effect of the residual conductivity. By denoting them with the same number plus a prime, they are sequentially:

- the linearized equation corresponding to charge conservation in the liquid

$$
\frac{M}{U^{1 / 2}} \frac{\partial q^{\prime}}{\partial t}+\frac{d Q_{0}}{d z} E_{z}^{\prime}+2 Q_{0} q^{\prime}+E_{0 z} \frac{\partial q^{\prime}}{\partial z}+\frac{M}{U^{1 / 2}} \frac{d Q_{0}}{d z} u_{z}+S q^{\prime}=0
$$

- the linearized boundary condition corresponding to conservation of electrical current across the interface

$$
\left[\frac{K}{K_{l}}\left(\frac{\epsilon}{\epsilon_{l}} E_{0 z} \nabla^{2} \phi^{\prime}+Q_{0} \frac{\partial \phi^{\prime}}{\partial z}\right)\right]_{0}+S \frac{\partial \phi^{\prime}}{\partial z}=0
$$

- the normal mode equation arising from charge conservation in the liquid

$$
\left(\frac{M}{U^{1 / 2}} \omega+2 Q_{0}+E_{0 z} \frac{d}{d z}+S\right)\left(\frac{d^{2}}{d z^{2}}-k^{2}\right) g+\frac{d Q_{0}}{d z} \frac{d g}{d z}-\frac{M}{U^{1 / 2}} \frac{d Q_{0}}{d z} u(z)=0,
$$

- the boundary condition for the normal modes corresponding to conservation of electrical current across the interface

$$
\left[\frac{K}{K_{l}}\left(\frac{\epsilon}{\epsilon_{l}} E_{0 z}\left(\frac{d^{2}}{d z^{2}}-k^{2}\right) g+Q_{0} \frac{d g}{d z}\right)\right]_{0}+S \frac{d g}{d z}=0
$$

- the final form of the normal mode equation arising from charge conservation in the liquid

$$
\left[E_{0 z} \frac{d^{3}}{d z^{3}}+\left(2 Q_{0}+S\right) \frac{d^{2}}{d z^{2}}+\left(\frac{d Q_{0}}{d z}-k^{2} E_{0 z}\right) \frac{d}{d z}-k^{2}\left(2 Q_{0}+S\right)\right] g(z)-\frac{M}{U^{1 / 2}} \frac{d Q_{0}}{d z} u(z)=0,
$$

- the normal mode equation arising from the equation of motion of the liquid

$$
\left(\frac{d^{2}}{d z^{2}}-k^{2}\right)^{2} u(z)+U k^{2}\left[E_{0 z} \frac{d^{2}}{d z^{2}}-\left(\frac{d Q_{0}}{d z}+k^{2} E_{0 z}\right)\right] g(z)=0,
$$


- the boundary condition corresponding to the jump of the electric field across the interface

$$
\epsilon_{r} \frac{d g}{d z}(0)+\frac{1}{L} \frac{d h}{d z}(0)=\left(\epsilon_{r} \frac{d E_{0}}{d z}(0+)-\frac{d E_{0}}{d z}(0-)\right) f_{0},
$$

- the final form of the boundary condition expressing conservation of electrical current across the interface

$$
\begin{gathered}
E_{0 z}(0+) \frac{d^{2} g}{d z^{2}}(0)+\left(Q_{0}(0+)+S\right) \frac{d g}{d z}(0)-k^{2} E_{0 z}(0+) g(0)- \\
\frac{K_{a}}{K_{l}}\left(\frac{E_{0 z}(0-)}{\epsilon_{r} L^{2}} \frac{d^{2} h}{d z^{2}}(0)-\frac{Q_{0}(0-)}{L} \frac{d h}{d z}(0)-k^{2} \frac{E_{0 z}(0-)}{\epsilon_{r}} h(0)\right)=0,
\end{gathered}
$$

- the boundary condition arising from the balance of normal electrical and mechanical stresses at the interface

$$
\begin{array}{r}
\frac{d^{3} u}{d z^{3}}(0)-3 k^{2} \frac{d u}{d z}(0)+k^{2} U\left(E_{0 z}(0+) \frac{d g}{d z}(0)+\frac{E_{0 z}(0-)}{\epsilon_{r} L} \frac{d h}{d z}(0)-\right. \\
\left.Q_{0}(0+) g(0)+Q_{0}(0-) h(0)\right) \\
+k^{2}\left(1-\frac{\rho_{a}}{\rho}+\frac{k^{2}}{\mathrm{Bo}}\right) g^{*} f_{0}=0
\end{array}
$$

\section{NUMERICAL SOLUTION AND RESULTS}

The boundary value problem formulated above is approached by utilizing a boundary value problem solver available from MATLAB, namely bvp4c. ${ }^{26}$ This MATLAB function implements a collocation method which uses cubic splines to approximate the solution.

The algorithm is particularly well suited for the computation of the dispersion relation $U(k)$, due to the possibility of taking advantage of a continuation technique. The method consists in using the solution obtained for a given value of $k$ as an initial guess for the solution corresponding to the next value of $k$. The continuation technique is also most useful when checking the robustness of the solution. The solution computed with a given error tolerance (a parameter that is chosen by the user) is used as the initial guess for a new computation with a more stringent error tolerance. If the results keep stable, one can be confident about the solution thus obtained.

For all the results presented in this section, the non-dimensional parameters correspond to a liquid layer of silicone oil with the following physical properties: $\epsilon / \epsilon_{0}=2.73$, kinematic viscosity $v$ $=50 \times 10^{-6} \mathrm{~m}^{2} / \mathrm{s}, \rho_{l}=960 \mathrm{~kg} / \mathrm{m}^{3}$, and $K_{l} / K_{a}=5 \times 10^{-6}$. Figure 2 is a plot of the critical value of the stability parameter $U$ as a function of the wavenumber $k$ in the case of no residual conductivity $(S=0)$. The liquid layer thickness is $1.2 \mathrm{~mm}$. The curve has only one minimum at $k=4.4$. This value of $k$ corresponds to a half-wavelength $\pi / k=0.714$, in non-dimensional units. Therefore, the size of the expected convective cells is of the order of the liquid layer thickness and corresponds to the experimentally observed instability due to the volume force. For the strong injection limit, Atten and Moreau ${ }^{5}$ found $k=4.35$ for the critical wavenumber in the case in which the injector was a free surface, and $\epsilon_{l} V_{l} /\left(K_{l} \eta\right)=95.15$ for the critical voltage, where $V_{l}$ is the voltage drop across the liquid layer. The instability threshold in Figure 2 corresponds to $U_{c}=0.1155$. Since the non-dimensional groups are formed taking as unit for voltage the total voltage drop across the extent of both layers, air and liquid, it is necessary to evaluate the corresponding voltage drop across the liquid layer. In doing so, using the hydrostatic solution of the electric potential, one obtains $\epsilon_{l} V_{l} /\left(K_{l} \eta\right)=94.25$, which compares favorably with the value given by Atten and Moreau. ${ }^{5}$

The model solved by Atten and Moreau ${ }^{5}$ neglected surface tension and gravity. Neither did they consider an air layer above the liquid, the injection taking place directly onto the liquid surface. Therefore, the results shown in Figure 2 indicate that surface tension and gravity do not influence the instability due to the volume force.

However, a novel feature is revealed when the liquid layer is decreased. A new minimum appears closer to $k=0$. This is shown in Figures 3-5. In Figure 3, the minimum is not yet visible, whereas 


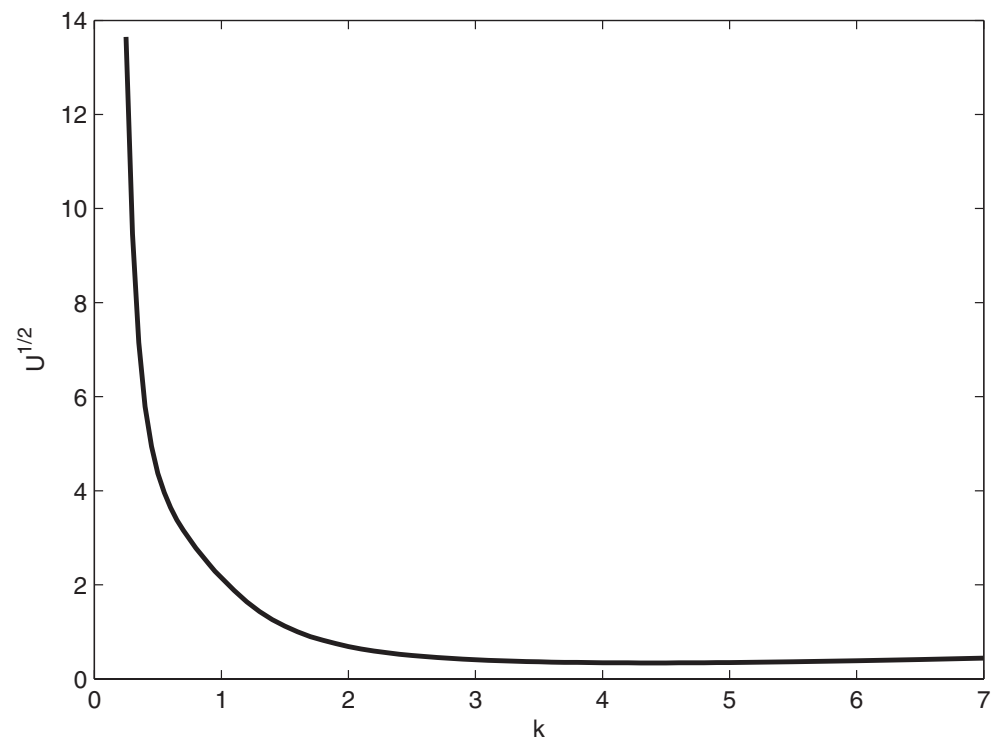

FIG. 2. Critical value of $\sqrt{U}$ versus $k$ for $S=0$. Other parameters are: $C=10, L=11.5$, Bo $=0.678, g^{*}=6.78, \epsilon_{r}=2.73$, $\rho_{a} / \rho=1.25 \times 10^{-3}, M=317.36$ and correspond to a layer of silicone oil of thickness $1.2 \mathrm{~mm}$ and kinematic viscosity $v=50 \times 10^{-6} \mathrm{~m}^{2} / \mathrm{s}$.

in the other two figures it is clearly noticeable. The new critical wavenumber is in the interval 0.5 $\leq k \leq 0.6$, which corresponds to a convective cell size almost an order of magnitude larger than the liquid layer thickness. Therefore, this new minimum must represent the rose-window instability.

Very remarkable is the behavior of the instability threshold for this new minimum, as a function of the liquid layer thickness. As it is observed in the figures, the instability parameter $U$ increases when $D$ decreases. This is shown in Figure 6, which is a plot of the predicted critical voltage as a function of the liquid layer thickness for silicone oil (for the liquid layer thickness $D=0.6 \mathrm{~mm}$, where a real minimum is not yet visible, the value of the voltage at the inflexion point has been

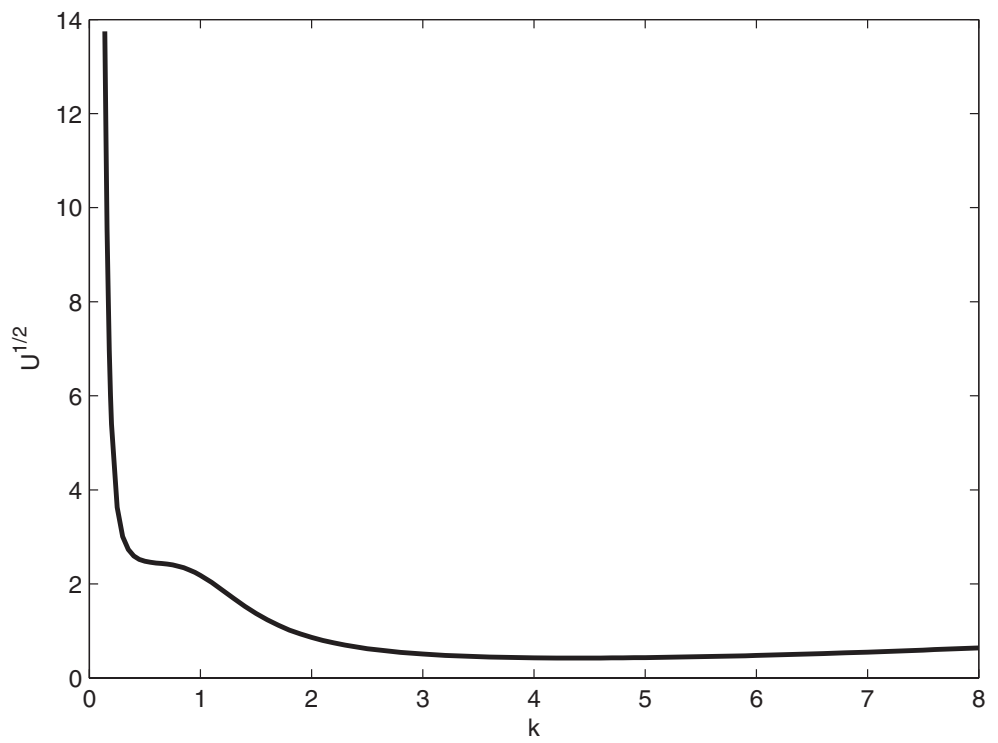

FIG. 3. Critical value of $\sqrt{U}$ versus $k$ for $S=0$. The non-dimensional parameters correspond to the same liquid as in Figure 2, but for a liquid layer thickness of $0.6 \mathrm{~mm}$. 


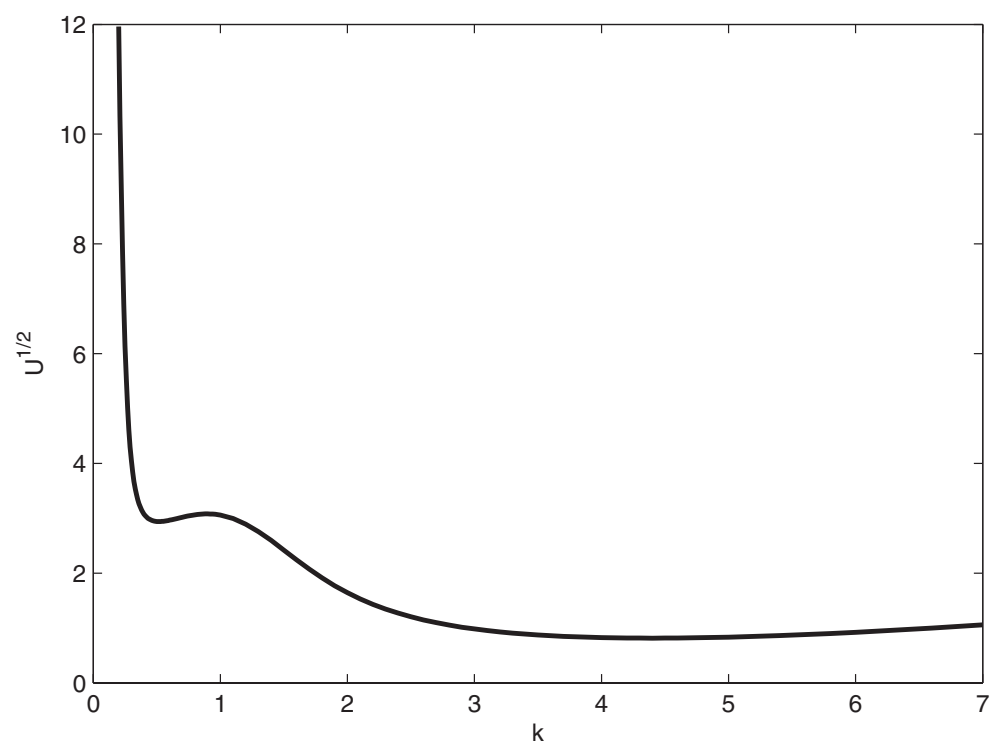

FIG. 4. Critical value of $\sqrt{U}$ versus $k$ for $S=0$. The non-dimensional parameters correspond to the same liquid as in Figure 2, but for a liquid layer thickness of $0.24 \mathrm{~mm}$.

taken). This plot is consistent with the experimental observations for non-Ohmic liquids, ${ }^{14}$ and in contrast with the experimental behavior ${ }^{14}$ as well as numerical results ${ }^{19}$ for Ohmic liquids.

On the other hand, these results question the validity of the simplified model, based on an infinite critical wavelength, presented in Refs. 16 and 17. This simplified model predicted a finite value of the critical voltage for zero wave-number, whereas the results presented here show that the critical voltage tends to infinity for $k \rightarrow 0$. Also the simplified model predicts that the instability threshold tends to infinity for a finite value of the liquid layer thickness, something that is not encountered here.

Figures 7-9 show the stability diagrams for a case of weak residual conductivity, $S=1$. The minimum in the curve $U(k)$ associated to the volume instability keeps occurring at a critical wavenumber $k$ in the interval $4.4 \leq k \leq 5$. However, the values of the critical parameter $U_{c}$, and

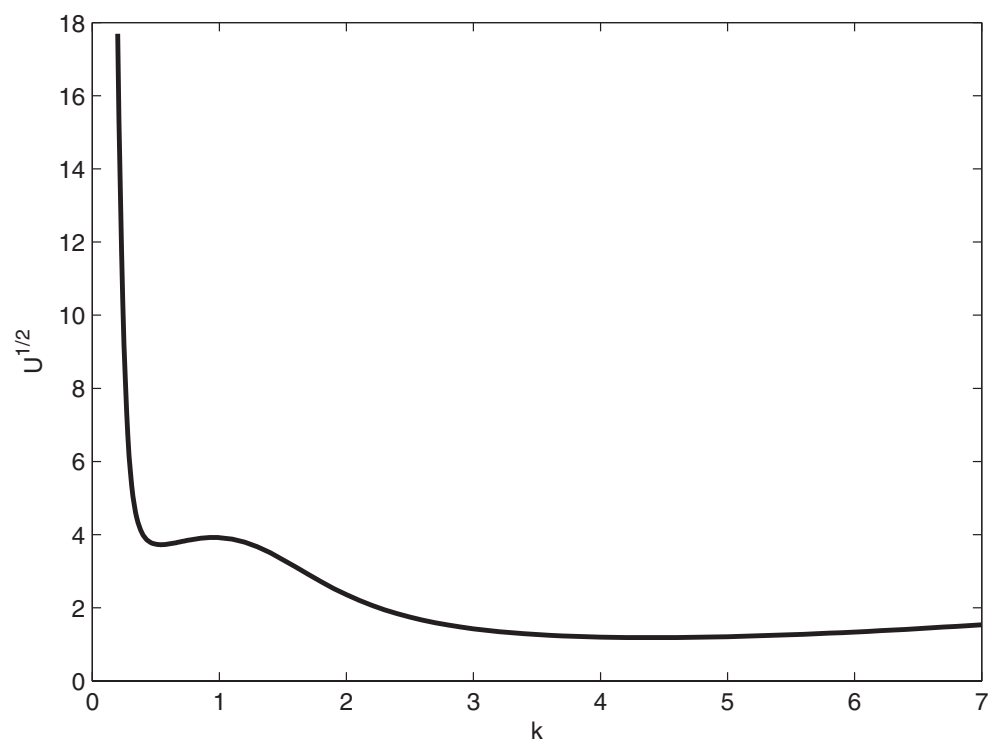

FIG. 5. Critical value of $\sqrt{U}$ versus $k$ for $S=0$. The non-dimensional parameters correspond to the same liquid than in Figure 2, but for a liquid layer thickness of $0.168 \mathrm{~mm}$. 


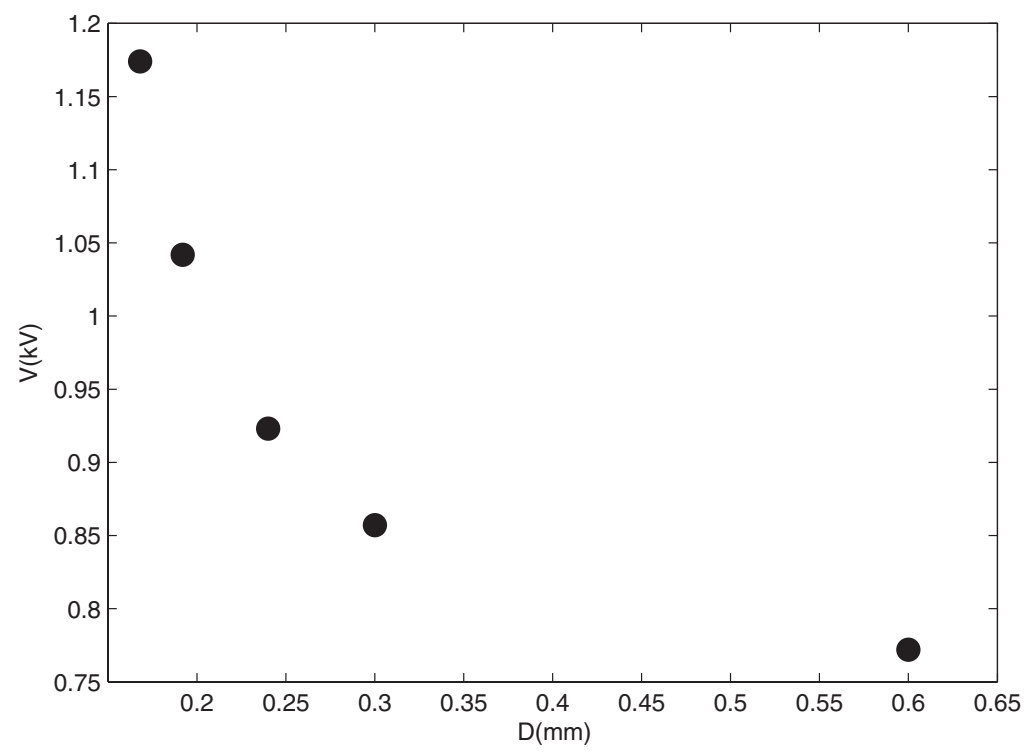

FIG. 6. Critical voltage as a function of the liquid layer thickness for silicone oil.

correspondingly of the critical voltage, are higher than for $S=0$. This is due to the fact that the conductivity contributes to the relaxation of the volume charge, thus decreasing the destabilizing volume force. This is also consistent with the experimental observations, where an increase in the conductivity of the sample leads to an increase of the voltage threshold and, eventually, to the disappearance of the instability.

Concerning the new minimum that appears at a low wavenumber, it becomes more noticeable for $S=1$. This may be attributed to the change in the charge distribution, which becomes more concentrated near the surface. In the analysis of the motionless state for the case of weak residual conductivity (Sec. III), it was shown that the charge distribution becomes a surface charge density for large values of $S$. And it was demonstrated in a previous work ${ }^{19}$ that in the Ohmic case, where the charge density is purely a surface one, the only minimum in the curve $U(k)$ has to be undoubtedly

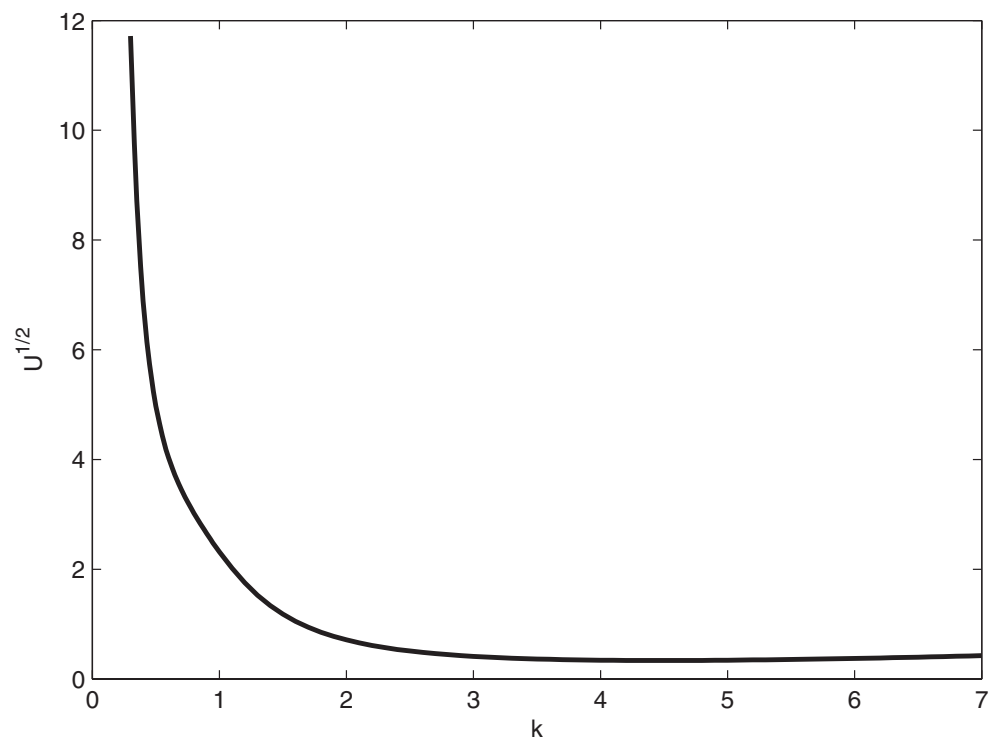

FIG. 7. Critical value of $\sqrt{U}$ versus $k$ for $S=1$ (model with residual conductivity). The other non-dimensional parameters and the liquid layer thickness are the same as in Figure 2. 


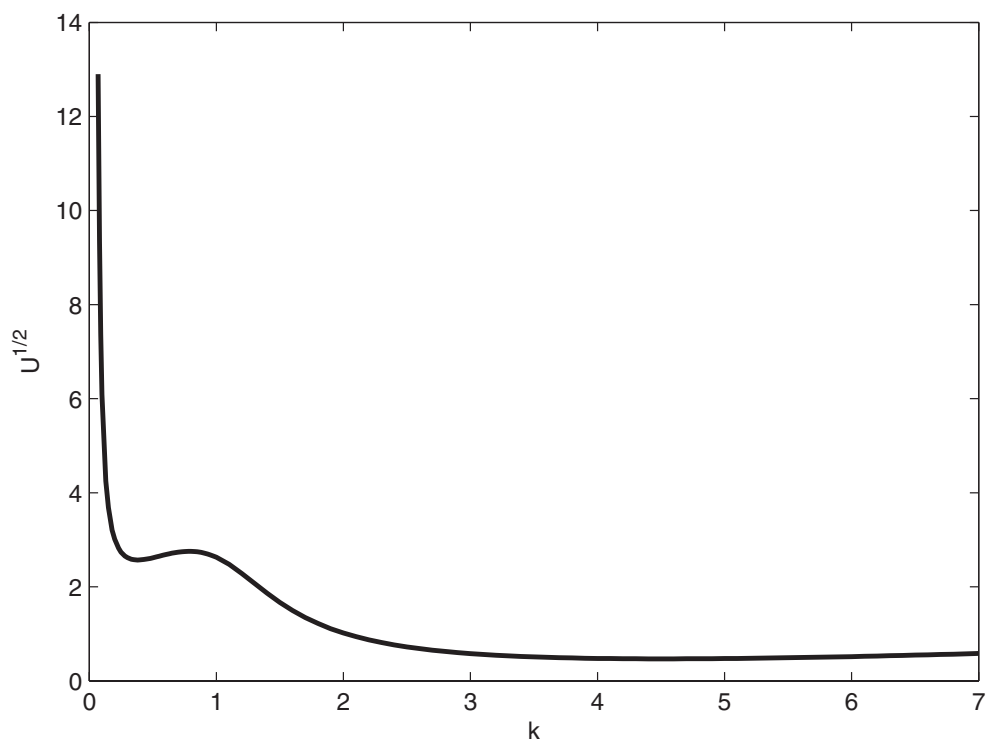

FIG. 8. Critical value of $\sqrt{U}$ versus $k$ for $S=1$. The non-dimensional parameters correspond to the same liquid as in Figure 7, but for a liquid layer thickness of $0.6 \mathrm{~mm}$.

associated to the surface charge instability. It is therefore reasonable to conclude that, when a residual conductivity is considered, the minimum becomes more noticeable due to the fact that the electrical forces act mainly upon the surface, and the situation is more similar to that of an Ohmic liquid.

The existence of two minima in the stability curves $U(k)$ opens the possibility of interchanging the order of appearance of the instabilities, depending on the liquid properties or the geometrical parameters. In all the curves presented here, the instability due to volume forces will appear first when the voltage is increased. Rose-window instability will follow. For Ohmic liquids, Rose-window instability may be present, if the conductivity is low enough. In these liquids, the volume instability is absent because there is no volume charge. Therefore, it is reasonable to think that an increase in the conductivity of the liquid will produce a situation where the Rose-window instability precedes the volume instability.

The perturbation of the charge density is a very rapidly varying function of the coordinate $z$ near the surface for the values of the parameters corresponding to Figures 7-9. These sharp gradients prevent us to obtain reproducible results for higher values of $S$ or smaller values of $D$ with the present numerical algorithm.

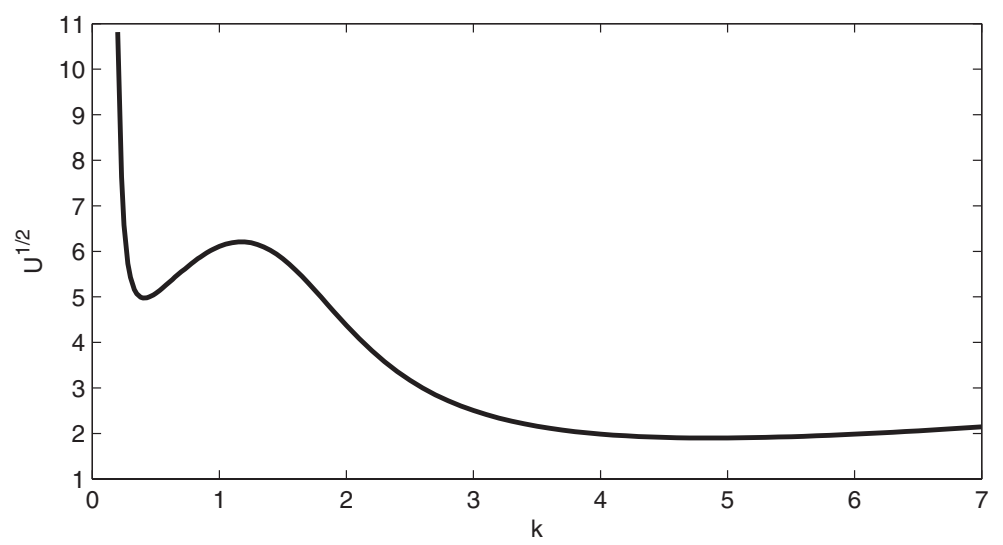

FIG. 9. Critical value of $\sqrt{U}$ versus $k$ for $S=1$. The non-dimensional parameters correspond to the same liquid as in Figure 7, but for a liquid layer thickness of $0.24 \mathrm{~mm}$. 
There are some experimental hints which suggest that the order of appearance of the instabilities might be reversed for an adequate choice of the geometrical parameters. Further experimental work would be needed to elucidate this point.

Figures 3-5 demonstrate that the same physical model of an insulating liquid that explains the volume instability may account for Rose-window instability. This is the most important finding of the present work.

\section{ACKNOWLEDGMENTS}

This work has been carried out with financial support from the Spanish Ministerio de Ciencia y Tecnología (MCYT) under Research Project No. FIS2011-25161, and Junta de Andalucía under Research Project Nos. P10-FQM-5735 and P09-FQM-4584.

${ }^{1}$ G. I. Taylor and A. D. McEwan, “The stability of a horizontal fluid interface in a vertical electric field," J. Fluid Mech. 22, 1 (1965).

2 J. R. Melcher, Field-Coupled Surface Waves (MIT Press, Cambridge, 1963).

${ }^{3}$ K. Mima, H. Ikezi, and A. Hasegawa, "Parametric excitation of collective modes in an electron layer on a liquid surface," Phys. Rev. B 14(9), 3953 (1976)

${ }^{4}$ R. W. Giannetta and H. Ikezi, "Nonlinear deformation of the electron-charged surface of liquid ${ }^{4} \mathrm{He}$," Phys. Rev. Lett. 47(12), 849 (1981).

${ }^{5}$ P. Atten and R. Moreau, "Stabilité électrohydrodynamique des liquides isolants soumis à une injection unipolaire," J. Méc. 11, 471 (1972)

${ }^{6} \mathrm{P}$. K. Watson, J. M. Schneider, and H. R. Till, "Electrohydrodynamic stability of space-charge-limited currents in dielectric liquids. II,” Phys. Fluids 13, 1955 (1970).

${ }^{7}$ J. M. Schneider and P. K. Watson, "Electrohydrodynamic stability of space-charge-limited currents in dielectric liquids. I. Theoretical study," Phys. Fluids 13, 1948 (1970).

${ }^{8}$ Electrohydrodynamics, edited by A. Castellanos (Springer, Vienna, 1998)

${ }^{9}$ C. S. Herrick, "Electroconvection cells in dielectric liquids interfaced with conducting fluids," Proc. R. Soc. London, Ser. A 336, 487 (1974).

${ }^{10}$ A. T. Ahmed El-Haddad, J. Fornazéro, and G. Mesnard, "Mécanismes de base de la déformation cellulaire d'une couche mince isolante liquide soumise à un flux de charges," Phys. Chem. Liq. 9, 201-218 (1980).

${ }^{11}$ B. Malraison and P. Atten, "Instabilité électrohydrodynamique due à l'injection d'ions à la surface libre d'un liquid isolant," J. Phys. III (France) 1, 1243-1249 (1991).

${ }^{12}$ A. T. Pérez, "EHD instabilities induced by corona discharge," in Proceedings of 12th International Conference on Dielectric Liquids (ICDL '96), Roma, Italy, 1996 (IEEE Press, Piscataway, NJ, 1996), pp. 126-129.

${ }^{13}$ A. T. Pérez, "Rose-window instability in low conducting liquids," J. Electrostat. 40-41, 141 (1997).

${ }^{14}$ F. Vega and A. T. Pérez, "Corona-induced electrohydrodynamic instabilities in low conducting liquids," Exp. Fluids 34, 726 (2003).

${ }^{15}$ P. Atten and D. Koulova-Nenova, "EHD instability of insulating liquids due to charge injection from the free surface," in Proceedings of 12th International Conference on Dielectric Liquids (ICDL '96), Roma, Italy, 1996 (IEEE Press, Piscataway, NJ, 1996), pp. 476-479.

${ }^{16}$ D. Koulova-Nenova and P. Atten, "EHD instability of air/liquid two layer system under unipolar charge injection," J. Electrostat. 40-41, 179 (1997).

${ }^{17}$ P. Atten, D. Koulova-Nenova, and A. Pérez, "On the EHD instability of a layer of insulating liquid subjected to unipolar charge injection due to corona discharge in air," in Proceedings of the International Workshop on Electrical Conduction, Convection and Breakdown in Fluids, Sevilla, 1998 (Universidad de Sevilla, Secretariado de Publicaciones, 1998), pp. 47-51.

${ }^{18} \mathrm{~F}$. Vega and A. T. Pérez, "Instability in a non-Ohmic/Ohmic fluid interface under a perpendicular electric field and unipolar injection," Phys. Fluids 14, 2738 (2002).

${ }^{19}$ R. Chicón and A. T. Pérez, "Instability of an interface between air and a low conducting liquid subjected to charge injection," Phys. Fluids 18, 104108 (2006).

${ }^{20}$ M. F. Schatz, S. J. VanHook, W. D. McCormick, J. B. Swift, and H. L. Swinney, "Onset of surface-tension-driven Bénard convection," Phys. Rev. Lett. 75, 1938 (1995).

${ }^{21}$ S. J. VanHook, M. F. Schatz, W. D. McCormick, J. B. Swift, and H. L. Swinney, "Long-wavelength instability in surfacetension-driven Bénard convection," Phys. Rev. Lett. 75, 4397 (1995).

${ }^{22}$ J. R. Melcher and G. I. Taylor, "Electrohydrodynamics: A review of the role of interfacial shear stresses," Annu. Rev. Fluid Mech. 1, 111 (1969).

${ }^{23}$ A. Castellanos and A. González, "Non-linear electrohydrodynamics of free surfaces," IEEE Trans. Dielectr. Electr. Insul. 5, 334 (1998).

${ }^{24}$ S. Chandrasekhar, Hydrodynamic and Hydromagnetic Stability (Dover, New York, 1981).

${ }^{25}$ J. Lighthill, Waves in Fluids (Cambridge University Press, Cambridge, 2002).

${ }^{26}$ J. Kierzenka and L. F. Shampine, "A BVP solver based on residual control and the Matlab PSE," ACM Trans. Math. Softw. 27(3), 299 (2001). 